\title{
Riskante Modernisierung: Wirkungen und Bewertungen der ERA-Einführung in Baden-Württemberg
}

Mit den neuen Entgeltrahmenabkommen (ERA) wurde in der Metall- und Elektroindustrie die Differenzierung in separate Lohn- und Gehaltssysteme für Arbeiter und Angestellte aufgehoben und damit eine Unterscheidung beseitigt, die als historisch überholt, dysfunktional und diskriminierend galt. Indes war die betriebliche Einführung nicht selten von heftigen Konflikten begleitet. Somit erschien es fraglich, ob die intendierten Gerechtigkeits- und Gleichbehandlungsziele erreicht und die angestrebte Verteilungsneutralität gewahrt werden würden. Inwieweit dies dennoch gelang und die Tarifparteien wie der Flächentarifvertrag insgesamt, wie erhofft, gestärkt werden konnten, diskutiert der Beitrag am Beispiel des baden-württembergischen ERA. ${ }^{1}$

\section{1 \\ Relevanz und politische Brisanz des ERA}

Das neue Entgeltrahmenabkommen (ERA) ist sowohl ein gesellschaftspolitisch bedeutsames als auch ein politisch brisantes Vorhaben. Im Kern handelt es sich bei ERA um ein verteilungspolitisches Projekt, bei dem allerdings nicht der Verteilungskonflikt zwischen Kapital und Arbeit, sondern die Differenzierung der Vergütung innerhalb der Arbeitnehmerschaft im Mittelpunkt stehen sollte. ERA zielt darauf, das Entgeltgefüge und damit die soziale Rangordnung in den Betrieben neu zu gestalten und zu begründen. Die Unterscheidung zwischen Arbeitern und Angestellten sollte fallen, Ungleichbehandlungen zwischen und innerhalb dieser Statusgruppen bei den Kriterien der Bewertung von Arbeit und Leistung sollten beseitigt und ein den derzeitigen Gerechtigkeitsvorstellungen entsprechendes, handhabbares und funktionales System geschaffen werden. Erklärte Absicht der Tarifparteien war es, Facharbeit aufzuwerten und die Entgeltlinie der Kaufleute auf die der Techniker anzuheben. Tätigkeiten unterhalb des Niveaus von Facharbeit sollten tarifvertraglich weitgehend gesichert sein. ${ }^{2}$

Neben einer verteilungspolitischen Funktion wird ERA eine große praktische und symbolische Funktion für die Stabi- lisierung der Flächentarifverträge zugesprochen, die bekanntermaßen seit geraumer Zeit unter Auszehrung leiden und auch dort, wo sie weiter gelten, die betrieblichen Arbeitsbedingungen nur noch eingeschränkt prägen (Bispinck/Bahnmüller 2007). Das gilt auch für die Entgeltrahmenabkommen. Die reale betriebliche Entgeltfindung hatte sich häufig weit von den modernisierungsbedürftigen Tarifregeln entfernt. Dem sollte mit ERA entgegengewirkt und die Leitfunktion des Tarifvertrags wieder hergestellt werden. Die Betriebe sollten wieder eine verlässliche Grundlage für die betriebliche Entgeltdifferenzierung erhalten und die Beschäftigten unmittelbar die Relevanz und den Nutzen des Flächentarifvertrags erfahren. Da die Tarifparteien, zumindest in BadenWürttemberg, ERA auch als Prüfstein ihrer eigenen Handlungs- und Reformfähigkeit verstanden, wurde der Umsetzungsprozess von beiden Seiten ausgesprochen aktiv betrieben und der Stellenwert für die Stabilisierung des Systems der Flächentarifverträge hoch angesetzt. Ein Misslingen der ERAUmsetzung wurde mit dem Ende des Flächentarifvertrags gleichgesetzt. ERA wurde zur Nagelprobe auf die Zukunftsfähigkeit des Flächentarifvertrags stilisiert (Bahnmüller/Schmidt 2007, 2008).

Eingriffe in die betriebliche Entgeltdifferenzierung und damit in das soziale Gefüge sind riskant. Die Tarifparteien wussten darum. In allen Tarifgebieten wurde deshalb vor Abschluss diskutiert, wie tief die
Eingriffe sein sollten, was man den Beschäftigten und was den Tarifverbänden zumuten konnte und wollte. Teilweise wählten die

\footnotetext{
1 Dargestellt werden Befunde eines von der HansBöckler-Stiftung geförderten Projekts zur Begleitforschung der ERA-Umsetzung in Baden-Württemberg. Empirische Basis sind auf der tariflichen Ebene 13 Expertengespräche sowie 57 Veranstaltungsteilnahmen, auf der betrieblichen Umsetzungsebene (teilweise mehrere) Expertengespräche in 25 Betrieben, standardisierte Beschäftigtenbefragungen mit 742 Teilnehmern in sechs Betrieben sowie eine schriftliche Befragung von Betriebsräten aus 284 Betrieben.

2 Eine Angleichung der Entgeltlinien von Kaufleuten und Technikern bedeutet nicht, dass alle Arbeitsaufgaben von Kaufleuten damit tariflich höher bewertet wären. Auch Abwertungen sind möglich und finden statt. Zudem sind die Veränderungen in den tariflichen Entgeltrelationen nicht mit den effektiven gleichzusetzen, da, wie wir in Abschnitt 2 noch genauer zeigen werden, die tarifliche $\mathrm{Pa}$ pierlage von der betrieblichen Entgeltrealität erheblich abwich.
}

Reinhard Bahnmüller, Dr., Geschäftsführer des Forschungsinstituts für Arbeit, Technik und Kultur (F. A. T. K.) an der Universität Tübingen. Arbeitsschwerpunkte: Industriesoziologie, Industrielle Beziehungen e-mail: reinhard.bahnmueller@unituebingen.de

Werner Schmidt, Dr., stellv. Geschäftsführer des Forschungsinstituts für Arbeit, Technik und Kultur (F. A. T. K.) an der Universität Tübingen. Arbeitsschwerpunkte: Industriesoziologie, Industrielle Beziehungen, Migrationsforschung. e-mail:w.schmidt@uni-tuebingen.de 
regionalen Tarifparteien bewusst eine risikoreiche Variante, teils wurde eine weniger risikobehaftete vorgezogen. Die Tarifparteien in Baden-Württemberg entschieden sich für den schwierigeren und auch riskanteren Weg. Alle Entgeltkomponenten wurden neu geregelt. Die Arbeitsbewertung, die bisher in den drei Tarifbezirken Baden-Württembergs auf unterschiedlicher Grundlage erfolgte, wurde tarifvertraglich durch ein komplett neues und einheitliches Verfahren abgelöst, das sogenannte Stufenwertzahlverfahren, einem Hybrid aus analytischer und summarischer Arbeitsbewertung, ergänzt und konkretisiert durch einen nunmehr verbindlichen Katalog tariflicher Niveaubeispiele. Belastungen, bisher Teil des Grundentgelts, wurden in - im Volumen von der jeweiligen Entgeltgruppe unabhängige - Zulagen verwandelt, die nicht mehr nur Gewerbliche erhalten können. Bezahlt werden Zulagen nur noch für Belastungen über einem „mittleren“ Niveau. Zudem wurde die Unterscheidung zwischen Leistungslohn, Zeitlohn und Gehalt aufgehoben und ein mit unterschiedlichen Methoden ermitteltes, doch in der Höhe einheitliches Leistungsentgelt für alle Beschäftigten eingeführt (zu Details vgl. IG Metall 2004/2006). Einig waren sich die Tarifparteien, dass eine nur oberflächliche Anpassung des alten an das neue System in Form einer Regelüberleitung ohne tatsächliche Neubewertung aller Arbeitsaufgaben vermieden werden sollte. ERA sollte zu einem wirklichen Neustart führen, der Eingriff sollte tief und nachhaltig sein.

Ob der riskante oder der weniger riskante Weg gewählt wurde, sicher war, es würde Gewinner und (relative) Verlierer unter den Beschäftigten geben. Die Tarifparteien trafen deshalb Vorkehrungen, um die Besitzstände von Beschäftigten, deren Verdienst vor ERA höher war als danach (sogenannte Überschreiter), abzusichern. In Baden-Württemberg wurde vereinbart, dass diese nominell kein Geld verlieren, ihnen allerdings maximal $10 \%$ des bisherigen Bruttoverdienstes bei künftigen Entgeltsteigerungen angerechnet werden können. Zudem wurde die bisherige betriebliche Entgeltsumme (für fünf Jahre) kollektiv abgesichert. Dennoch blieb ein Verdienstrisiko für den Einzelnen und in Abhängigkeit von der Anzahl der Überschreiter auch ein Akzeptanzrisiko für ERA und die Kollektivakteure - nicht zuletzt die IG Metall.
Die Risiken der Arbeitgeber wurden durch Zusicherung einer fünfjährigen Kostenneutralität abgesichert. Kostenneutralität war als Korridor definiert. Dieser umfasste den Bereich von Null bis 2,79\% an Mehrkosten. Um darüber hinausgehende Kosten abzudecken, wurden in drei Lohnrunden Teile der dort vereinbarten Entgelterhöhungen nicht ausbezahlt, sondern in einen betrieblichen ERA-Anpassungsfonds abgeführt. Sollte ERA teurer werden als die prognostizierten 2,79 \%, können die Arbeitgeber Mehrkosten durch Kürzungen an anderer Stelle wieder ausgleichen. Umgekehrt darf die Entgeltsumme der Betriebe durch ERA in diesem Zeitraum auch nicht unter das bisherige Niveau fallen (Nulllinie), d.h. fünf Jahre lang können die Arbeitgeber auch bei einem für sie günstigen ERA-Ergebnis keine Kosten sparen. Die erwarteten Mehrkosten durch ERA von 2,79\% wurden durch Modellrechnungen ermittelt. ${ }^{3}$ Die erwarteten ERA-Kosten waren somit durch im Rahmen "normaler" Lohn- und Gehaltstarifrunden vereinbarte Entgeltsteigerungen vorfinanziert.

Die Regelungen zur Kostenneutralität sollten den Verteilungskonflikt zwischen Kapital und Arbeit ausklammern und die betrieblichen Verhandlungsprozesse auf die gemeinsame Herstellung einer neuen, tarifvertraglich fundierten Entgeltordnung ausrichten: integratives, nicht distributives Bargaining war intendiert (Walton/McKersie 1965). Im Verteilungsverhältnis von Kapital und Arbeit sollte die Reform insgesamt neutral ausfallen.

\section{2 \\ Entgeltpolitische Effekte}

Welche entgeltpolitischen Wirkungen von ERA lassen sich nun nach Abschluss des offiziellen Einführungszeitraumes feststellen? Konstatieren lässt sich zunächst: Die ERA-Regelungen sind breit diffundiert. Es gab zwar zum Ende der Einführungsfrist (Februar 2008) etliche Betriebe, die ERA noch nicht angewendet und einen Antrag auf Fristverlängerung gestellt hatten, in jenen ca. 500 Unternehmen mit vollzogener Einführung arbeiteten jedoch etwa $80 \%$ der Beschäftigten der Mitgliedsfirmen von Südwestmetall. Zwischenzeitlich sind weitere Betriebe hinzugekommen und ERA ist aktuell für etwa $90 \%$ der Beschäftigten in tarifgebundenen Betrieben in Kraft. Da je- doch in tarifgebundenen Betrieben lediglich $56 \%$ der Beschäftigten der gesamten Branche arbeiten, gilt ERA gleichwohl nur für die Hälfte aller Beschäftigten.

Wirkung zeigt ERA nicht nur in der Breite, sondern auch in der Tiefe: Es gibt kaum tarifgebundene Betriebe, in denen ERA nicht deutliche Veränderungen der betrieblichen Entgeltstrukturen bewirkt hätte. Es kam auf breiter Front zu Auf- wie zu Abwertungen von Arbeitsaufgaben. Die betriebliche Entgeltfindung ist in einem seit Langem nicht mehr gekannten Maße tariflich geprägt. Es ist somit gelungen, die Relevanz von Tarifverträgen wieder spürbar und nachvollziehbar zu machen - auch für jene, die Tarifverträgen bisher keine Bedeutung für ihre Entgeltfindung zubilligten. Relevanz und Akzeptanz von ERA gehen jedoch, wie wir zeigen werden, nicht Hand in Hand. Die betrieblichen Resultate der ERA-Einführung fielen nicht immer so aus, wie die Betriebsräte, die Beschäftigten und mitunter auch die Arbeitgeber es sich wünschten.

Die ERA-Umsetzung verlief in BadenWürttemberg konfliktreicher als erwartet, teils weil die Arbeitgeber im Widerspruch zur vereinbarten Kostenneutralität ERA zur Kosteneinsparung nutzten, teils wegen gewollter und tariflich vereinbarter Veränderungen in der Wertigkeit von Tätigkeiten. Und nicht zuletzt, weil die betriebliche Entlohnungspraxis aus verschiedenen Gründen mitunter weit oberhalb des tariflichen Mindestniveaus lag: Die betriebliche Entgeltpolitik der Betriebsräte hatte daran Anteil. Ebenso verantwortlich waren Angebotsengpässe auf dem Arbeitsmarkt, die Anerkennung von Loyalität und besonderen Leistungen durch Vorgesetzte sowie der verbreitete Verzicht auf Abgruppierungen bei Rationalisierungsprozessen. Gleichwohl galten diese Verdienste als tariflich gedeckt. Die Diskrepanz zwischen als tariflich gesichert geltendem und definitiv gesichertem Entgelt wurde bei der Neueingruppierung im Zuge der ERA-Einführung erst in vollem Umfang sichtbar. Die tarifsystematisch vereinbarten Effekte werden deshalb

\footnotetext{
3 Die 2,79 \% entsprechen dem erwarteten durchschnittlichen Delta zwischen den Systemkosten von ERA und den bisherigen Systemkosten. Auf welcher Basis der Systemkostenvergleich stattfindet, ist allerdings in den regionalen ERA-Abkommen unterschiedlich definiert, was Vergleiche erschwert. Teilweise erfolgt die Berechnung auf der Basis der Tarifentgelte (Niedersachsen), teils der Effektiventgelte (Baden-Württemberg).
} 


\section{Abb. 1: Höher bewertete Arbeitsaufgaben durch ERA im Urteil der

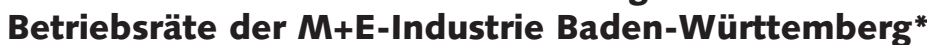 \\ - in \% der Betriebe mit Angabe "trifft zu" -}

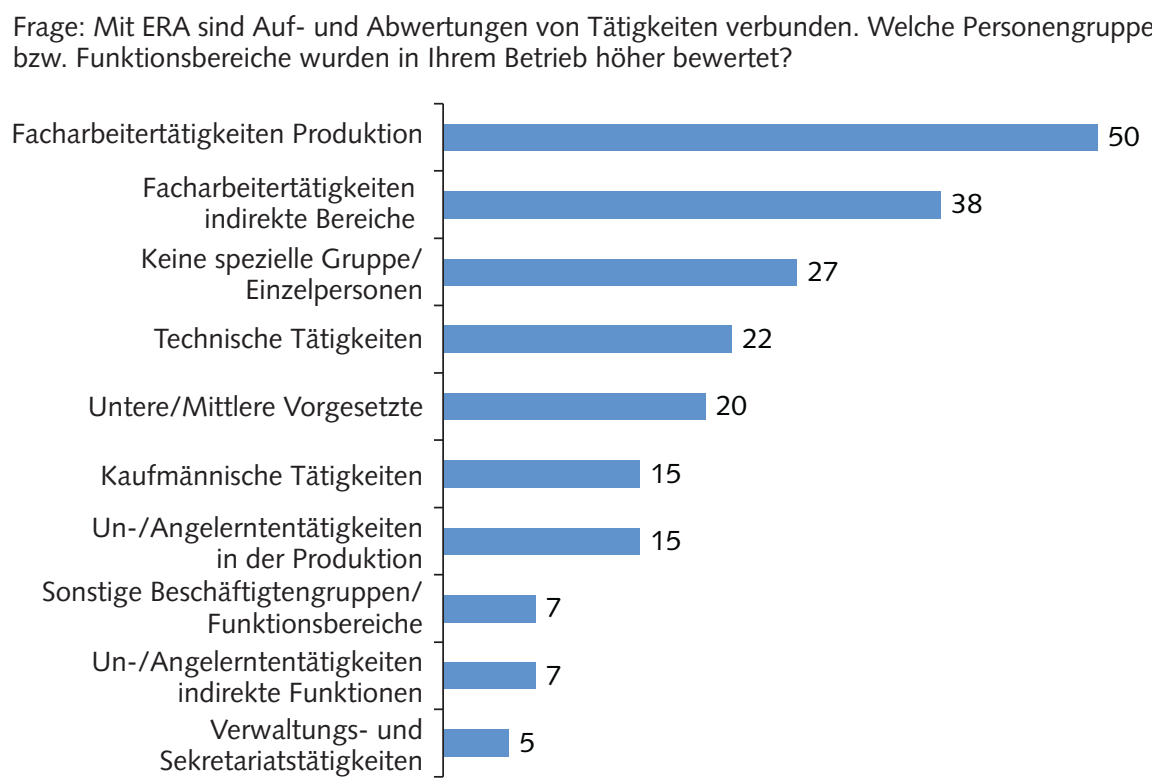

* Betriebsrätebefragung; Betriebe mit eingeführtem ERA, $n=149$. Quelle: Darstellung der Autoren.

WSI MITEELUNGEN

von jenen überlagert, die sich aus den Abweichungen zwischen der tariflichen $\mathrm{Pa}$ pierlage und der tatsächlichen betrieblichen Vergütung ergeben. In der Folge können selbst Beschäftigte mit tarifsystematisch aufgewerteten Arbeitsaufgaben faktisch niedriger bewertet werden, tarifsystematische „ERA-Gewinner“ zu effektiven „ERA-Verlierern“ werden.

Wir konzentrieren uns hier auf die effektiven Folgen, die tatsächlichen Auf- und Abwertungen, die Besetzung der Entgeltgruppen (EG) sowie die Überschreiterquote bzw. den Anteil der Unterschreiter (Verdienst war vor ERA niedriger).

\subsection{AUF- UND ABWERTUNGEN}

Mit Blick auf die Neubewertung der Arbeitsaufgaben zeigt unsere Befragung von Betriebsräten, ${ }^{4}$ dass die intendierte Höherbewertung der Facharbeit in der Produktion und in produktionsnahen Bereichen in einem Großteil der Betriebe erreicht wird. Sofern die höher bewerteten Facharbeitertätigkeiten spezifiziert werden, handelt es sich vornehmlich um solche im Werkzeugbau, in der Instandhaltung, um (Werkstatt-)Programmierer, Einrichter, Anlagenführer und junge Facharbeiter. Teilweise profitieren auch qualifizierte technische Angestellte, insbesondere (Entwick-
lungs-)Ingenieure, Konstrukteure, qualifizierte Techniker. Ähnlich häufig erfahren untere bzw. mittlere Führungskräfte eine Aufwertung (20\% der Betriebe), etwa Vorarbeiter, Gruppenführer, Meister oder Schichtführer. Zudem gibt es Aufwertungseffekte für Funktionen oder Personen, die keiner speziellen Gruppenlogik folgen. Von einer Aufwertung kaufmännischer Tätigkeiten berichten nur wenige Betriebsräte (15\%), zu höheren Bewertungen von Unund Angelernten in indirekten Funktionen sowie von Verwaltungs- und Sekretariatstätigkeiten kommt es fast nirgends (Abbildung 1).

Den Aufwertungen stehen Abwertungen in beträchtlichem Umfang gegenüber (Abbildung 2). Letztere betreffen am häufigsten zwei Gruppen: Verwaltungs- und Sekretariatstätigkeiten wurden in $73 \%$ und kaufmännische Funktionen in $69 \%$ der Betriebe abgewertet. Signifikante Unterschiede nach Branchen finden sich keine. Die Abwertung von Sekretariatstätigkeiten ist in größeren Betrieben deutlich stärker ausgeprägt (>1000 Besch. $95 \%,<100$ Besch. $44 \%)$. Bei kaufmännischen Tätigkeiten ist die Betriebsgröße nicht relevant.

Mit den technischen Tätigkeiten sowie mit solchen von Angelernten in der Produktion und etwas seltener in den indirekten Bereichen treffen die Abwertungen zwei weitere Gruppen. Technische Tätigkeiten sind vor allem dann betroffen, wenn sich die fachlichen Anforderungen aufgrund computerunterstützten Arbeitens nachhaltig verändert hatten (Konstrukteure, technische Zeichner) oder Engpässe zu hohen Marktpreisen auf dem externen Arbeitsmarkt führten. Dazu zählen etwa ITTätigkeiten, die zwar ihren hohen Marktwert wieder eingebüßt hatten, bei denen jedoch dieser Abwertungsprozess intern erst mit ERA nachvollzogen wurde. Auch bei unteren und mittleren Vorgesetzten wird häufiger von Abwertungen als von Aufwertungen berichtet $(30 \%: 20 \%)$.

Betroffen von Abwertungen sind zudem Beschäftigte, die bisher als Facharbeiter galten, so auch bezahlt wurden, ohne formell über einen entsprechenden $\mathrm{Ab}$ schluss zu verfügen. Hier ist ein sich künftig wahrscheinlich noch verschärfender Streit darüber im Gang, was Facharbeit ist und wie sie bezahlt wird. Insgesamt gesehen gab es Abwertungen somit oberhalb, vor allem aber im Grenzbereich von Facharbeit (EG 7) und darunter. Letztlich deuten unsere Ergebnisse darauf hin, dass ,einfache“ Arbeit vielfach billiger wird - zwar nicht sofort, aber mittelfristig.

\subsection{VERHÄLTNIS VON "ERA-GEWINNERN“ ZU "ERA-VERLIERERN“}

Der Saldo der Auf- und Abwertungen findet seinen Niederschlag in der Besetzung der Entgeltgruppen. Unseren Befunden zufolge befanden sich zum Jahresende 2007/2008 $32 \%$ der Beschäftigten unterhalb der Eckentgeltgruppe 7, 39 \% im Bereich EG 7 bis EG 12 (traditioneller Facharbeiterbereich) und $27 \%$ darüber (EG 13 - EG 17). Verglichen mit den Besetzungen vor ERA ist der Anteil der Beschäftigten, die unterhalb der Eckentgeltgruppe eingruppiert sind, deutlich gestiegen. Exakt

\footnotetext{
4 Die nachfolgenden statistischen Befunde stützen sich auf eine 2007/2008 durchgeführte schriftliche Befragung von Betriebsräten aller tarifgebundenen Betriebe in Baden-Württemberg. Ausgegeben wurden 1082 Fragebogen, der Rücklauf betrug 284 (26\%), darunter 149 aus Betrieben mit eingeführtem ERA, 135 aus Betrieben mit zum Zeitpunkt der Befragung noch nicht eingeführtem. Der Anteil der Beschäftigten des Befragungssamples an allen Beschäftigten in tarifgebundenen Betrieben liegt bei $59 \%$, derjenige der Betriebe mit erfolgter Einführung bei 43 \% (vgl. hierzu auch Bahnmüller/Schmidt 2008).
} 
lässt sich dies nicht bestimmen. Näherungsweise ist davon auszugehen, dass sich der Anteil um etwa 10 Prozentpunkte erhöht hat. ${ }^{5}$

Erst die Summe der Veränderungen bei der Arbeits-, der Belastungs- und der Leistungsbewertung bestimmt, ob ein Beschäftigter zum Überschreiter wird, dessen Verdienst vor ERA höher war, oder zum Unterschreiter mit vor ERA geringerem Verdienst. Nimmt man das Verhältnis von Überschreitern und Unterschreitern als Maßzahl, gibt es mehr „ERA-Verlierer“ als „ERA-Gewinner". Im betrieblichen Durchschnitt liegt der „Überschreiteranteil“ bei $54 \%$, bezogen auf die Beschäftigten bei 59 \%. Konzentriert sind die Überschreiter in den Verwaltungs- und Sekretariatsbereichen, bei den kaufmännischen Angestellten, bei den Geringqualifizierten in der Produktion (vor allem ehemalige „Leistungslöhner"), aber auch bei qualifizierten technischen Angestellten. Ältere Beschäftigte gehören ebenfalls recht häufig zu den Überschreitern.

\subsection{VERTEILUNGSPOLITISCHE EFFEKTE}

Nicht nur in der Perspektive des Anteils von Überschreitern und Unterschreitern sind die Wirkungen der ERA-Einführung aus Arbeitnehmersicht problematisch, sondern auch hinsichtlich der verteilungspolitischen Effekte zwischen Kapital und Arbeit. Auskunft über die diesbezüglichen mittel- und langfristigen Wirkungen von ERA gibt das Delta der Systemkosten alt zu neu, sofern alle Ausgleichsbeträge, d.h. die „Übergangskosten“ von der alten in die neue Welt, unberücksichtigt bleiben. Verteilungsneutral wäre die ERA-Einführung, wenn dabei im Durchschnitt der Betriebe die in früheren Tarifrunden bereits finanzierten 2,79\% an Mehrkosten erreicht würden. Diese Marge wird in Baden-Württemberg verfehlt. Wo die Systemkosten tatsächlich liegen, ist schwer zu ermitteln. Vielen Betriebsräten ist diese Kennzahl lediglich näherungsweise bekannt. Berücksichtigt man geschätzte und exakte Werte, werden unserer Betriebsrätebefragung zufolge die Personalkosten in $4 \%$ der Betriebe dauerhaft oberhalb der Marge von $2,79 \%$ liegen, in $54 \%$ im Bereich zwischen $1 \%$ und $2,79 \%$, in $11 \%$ zwischen $0 \%$ und $1 \%$ und in einem Viertel unter dem bisherigen Niveau. Es gibt eine Reihe von Hinweisen, dass der Anteil der Betriebe unter der Null-Linie tatsächlich eher größer sein

\section{Abb. 2: Niedriger bewertete Arbeitsaufgaben durch ERA im Urteil der Betriebsräte der $\mathbf{M}+\mathbf{E}-$ Industrie Baden-Württemberg* \\ - in \% der Betriebe mit Angabe "trifft zu" -}

Frage: Mit ERA sind Auf- und Abwertungen von Tätigkeiten verbunden. Welche Personengruppen bzw. Funktionsbereiche wurden in Ihrem Betrieb niedriger bewertet?

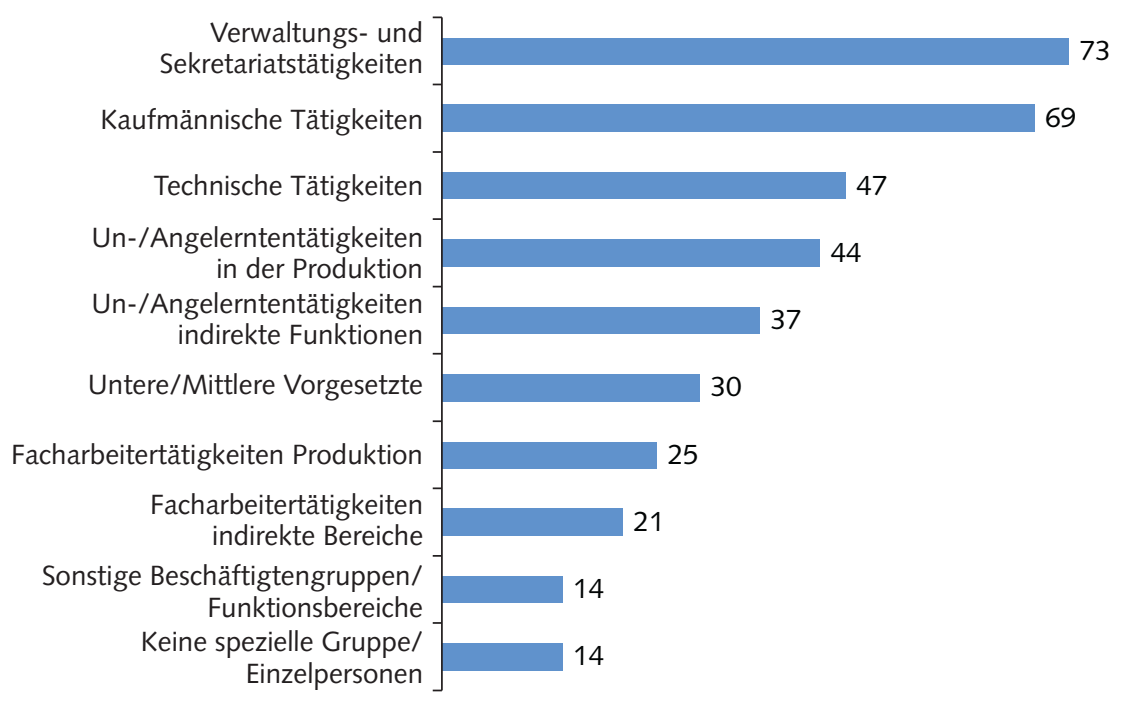

Betriebsrätebefragung; Betriebe mit eingeführtem ERA, $n=149$. Quelle: Darstellung der Autoren.

WSI

MITTEILUNGEN

könnte. So rechnet z. B. die Hälfte der Betriebsräte bei direkter Nachfrage mit einer dauerhaften Absenkung des betrieblichen Entgeltniveaus (Tabelle 1), soweit in einzelnen Fällen die Angaben zu den Kosten überprüft werden konnten, erwiesen sie sich partiell als zu hoch. Bisweilen wurden offenbar die Systemkosten mit den höheren betrieblichen Kosten, in die Ausgleichszahlungen an Über- bzw. Unterschreiter mit eingehen, verwechselt. Läge das Delta der Systemkosten im Durchschnitt unter Null, müsste man auf jeden Fall davon sprechen, dass ERA nicht kurz-, aber mittelfristig zu einer Kosteneinsparung führt. Liegt es über Null, doch deutlich unter $2,79 \%$, dann gilt insofern dasselbe, als die intendierte (und finanzierte) Verteilungsneutralität nicht erreicht wäre. So oder so muss deshalb konstatiert werden: Die Verteilungsrelationen sind, sofern keine weiteren Korrekturen erfolgen, im Ergebnis zwar nicht kurz-, aber mittelfristig zulasten der Beschäftigten verschoben.

\subsection{GLEICHBEHANDLUNGS- UND GERECHTIGKEITSZIELE}

Besser sieht die Bilanz hinsichtlich der mit ERA verfolgten Gleichbehandlungs- bzw. synchron vergleichenden Gerechtigkeitsziele aus, also bei der Beseitigung der Sta- tusdifferenzierung zwischen Arbeitern und Angestellten, bei der Aufhebung von Ungerechtigkeiten bei der Bezahlung von Beschäftigten mit vergleichbaren Arbeitsanforderungen, der ungleichen Vergütung von Belastungen sowie ungleichen Verdienstchancen im Leistungsentgelt bei vergleichbaren Leistungen. Die erfolgreiche Beseitigung all dieser Effekte wird von einer absoluten Mehrheit der Betriebsräte attestiert. Auch die Aufwertung von Facharbeit als Ergebnis der Gleichbehandlung gehört dazu (Tabelle 1). Andererseits widersprechen auch bis zu $27 \%$ der Betriebsräte explizit. Dennoch: Die synchronen Ge-

\footnotetext{
5 Ein Vorher-Nachher-Vergleich birgt methodische Probleme. Die hinter den bisherigen Lohn- und Gehaltsgruppen bzw. den neuen Entgeltgruppen steckenden Arbeitsanforderungen lassen sich überwiegend nicht unmittelbar vergleichen, lediglich die Eckentgeltgruppe 7, die in der Regel eine einschlägige berufliche Ausbildung voraussetzt, eignet sich als Bezugspunkt. Solche Eckentgeltgruppen gab es mit der LG 7/AW 6 für Gewerbliche und K2, T2 für Angestellte auch im Altsystem. Da Belastungen im Altsystem Teil des Grundentgelts waren und sie mit ERA als Zulagen ausgegliedert sind, wird der Vergleich zusätzlich erschwert. Die dadurch systembedingt ausgelöste Drift nach unten bei der Entgeltgruppenbesetzung bei gewerblichen Beschäftigten wurde in unserer schätzung berücksichtigt.
} 


\begin{tabular}{|c|c|c|c|c|}
\hline $\begin{array}{l}\text { Tabelle1: Entgeltpolitische Effekte } \\
\text { Betriebsräte* - in \% - }\end{array}$ & On ER & m Urte & der & \\
\hline Frage: Welche relevanten Effekte haben sich in Ihre & Betrieb du & rch die ERA- & inführung er & seben? \\
\hline & (Eher) ja & Teils, teils & (Eher) nein & $\begin{array}{l}\text { Weiß nicht/ } \\
\text { offen }\end{array}$ \\
\hline $\begin{array}{l}\text { Realisierung des Grundsatzes: Gleicher Geldbetrag } \\
\text { für gleiche Belastung }\end{array}$ & 61 & 8 & 19 & 12 \\
\hline $\begin{array}{l}\text { Herstellung gleicher Verdienstchancen für } \\
\text { vergleichbare Leistung }\end{array}$ & 58 & 30 & 10 & 1 \\
\hline $\begin{array}{l}\text { Beseitigung von Ungerechtigkeiten in der } \\
\text { Eingruppierung von Arbeitern und Angestellten }\end{array}$ & 55 & 21 & 23 & 1 \\
\hline $\begin{array}{l}\text { Transparente und nachvollziehbare Ordnung in der } \\
\text { Entlohnung }\end{array}$ & 54 & 21 & 22 & 2 \\
\hline Aufwertung der Facharbeit & 54 & 18 & 27 & 1 \\
\hline $\begin{array}{l}\text { Dauerhafte Absenkung des betrieblichen } \\
\text { Entgeltniveaus }\end{array}$ & 51 & 19 & 29 & 1 \\
\hline Entgeltverluste für Beschäftigte & 44 & 27 & 29 & 1 \\
\hline Neue Ungerechtigkeiten in der Entlohnung & 35 & 25 & 39 & 1 \\
\hline Abwertung von Facharbeit & 21 & 16 & 63 & 1 \\
\hline $\begin{array}{l}\text { *Betriebsrätebefragung; ERA eingeführt, } n=149 \\
\text { Quelle: Zusammenstellung der Autoren. }\end{array}$ & & & 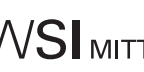 & EILUNGEN \\
\hline
\end{tabular}

rechtigkeitsziele, auf die sich die Tarifparteien bei ERA verständigt haben, sind vielfach erreicht. Sie stehen jedoch in einem Spannungs- und Konkurrenzverhältnis zu alternativen Gerechtigkeitsvorstellungen von Beschäftigten (und auch Betriebsräten), die sich auch auf lebens- bzw. berufsgeschichtliche (diachrone), qualifikationsbezogene oder reproduktions- bzw. bedürfnisorientierte Dimensionen beziehen. Auch diese beeinflussen die ERA-Bewertung und prägen das Urteil insbesondere der Beschäftigten (vgl. Abschnitt 3). Das Gleichheitsprojekt ERA hat zudem einige andere Schrammen. Nicht unerhebliche Ungleichbehandlungen gibt es zwischen den abgesicherten ,alten“" und den neu eingestellten Beschäftigten. Der Abstand ist teilweise beträchtlich, kann $15 \%$ und mehr betragen. In manchen Betrieben wurden allerdings auch kollektive Besitzstandssicherungen vereinbart, von denen auch neu blem etwas entschärft wird. Eingestellte profitieren, wodurch das Pro-

\section{Abb. 3: Gesamtbewertung des ERA durch Betriebsräte, Beschäftigte (aus Sicht der Betriebsräte) sowie Beschäftigte (Direktbefragung)* - in \% -}

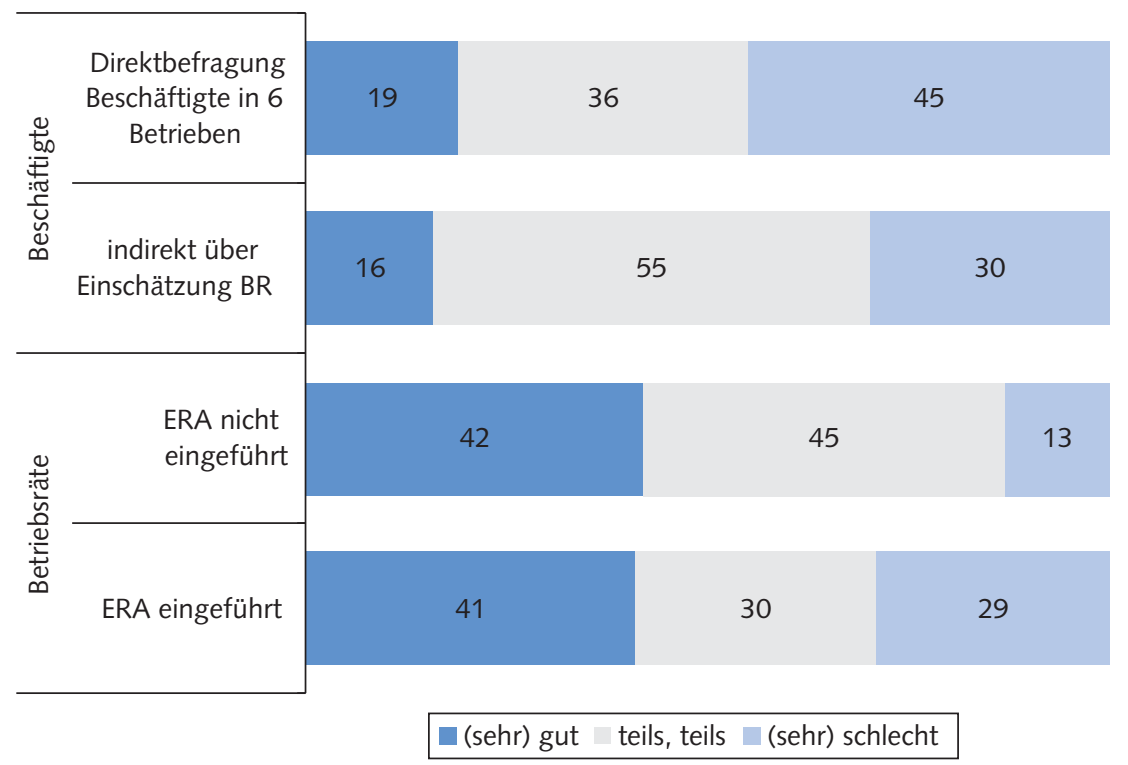

*Betriebsrätebefragung; ERA eingeführt $n=149$, ERA nicht eingeführt

fragung $n=74$

Quelle: Darstellung der Autoren.
WSI MITELLUNGEN

\section{ERA-Bewertung und Anerkennungseffekte}

Angesichts dieser Befunde darf es nicht wundern, wenn die ERA-Bewertung der Betriebsräte eher durchwachsen ausfällt (Abbildung 3). In Betrieben mit eingeführtem ERA $(\mathrm{n}=149)$ wird ERA von $41 \%$ mit "gut" oder in wenigen Fällen mit, ,sehr gut" bewertet, $29 \%$ bewerten ERA „schlecht“ oder „sehr schlecht", $30 \%$ sehen sowohl negative als auch positive Aspekte und antworten mit „teils, teils“. Die Bewertung ist unabhängig von Branche und Betriebsgröße, geprägt wird sie in starkem Maße durch die betriebliche „Überschreiterquote", die durch die Aktivität des Betriebsrats und der Belegschaft allerdings selbst mit beeinflusst werden kann, und den Grad an Kooperation des Arbeitgebers im Einführungsprozess. Je kooperativer der Einführungsprozess verläuft, desto positiver wird ERA beurteilt. Ein dennoch hoher Anteil an Überschreitern setzt solchen positiven Erfahrungen allerdings klare Grenzen. Die ERA-Bewertung schwankt zudem im Zeitverlauf. Von Betriebsräten in Unternehmen, die ERA bereits in den ersten beiden Jahren eingeführt haben, wird ERA kritischer bewertet als von solchen, die aus den Erfahrungen anderer lernen und sich auf das offensive Vorgehen der Arbeitgeber in Allianz mit Südwestmetall einstellen konnten (vgl. dazu Bahnmüller/Schmidt 2007, 2008). Gelingt es den Betriebsräten zudem, im betrieblichen Verhandlungsprozess deutliche Verbesserungen zu erreichen, kann sich das anfänglich negative Urteil auch ins Positive wenden. Die nicht hintergehbare Größe bleibt jedoch die Überschreiterquote. Je geringer sie ausfällt bzw. je stärker sie gedrückt werden kann, desto besser wird ERA bewertet. Nur in Ausnahmefällen wird ERA auch bei hoher Überschreiterquote aufgrund kooperativer Einführungspraxis der betrieblichen Akteure, guter Kommunikationsstrategie und optimaler Steuerung von Ausschüttungen aus dem ERA-Anpassungsfonds von Betriebsräten insgesamt positiv bewertet.

Schlechter als die Bewertung der Betriebsräte fällt die der Beschäftigten aus. Sie bewerten ERA und die ERA-Einführung überwiegend negativ. Sowohl die direkte schriftliche Befragung von Beschäftigten in sechs Betrieben (Abbildung 3) als auch die 
Auskünfte der Betriebsräte in der Flächenbefragung sowie die Expertengespräche bestätigen dieses Ergebnis. Die negative ERABewertung der Beschäftigten erklärt sich nicht zuletzt durch die hohe Überschreiterquote. Beschäftigte, die zu den Überschreitern zu zählen sind, bewerten ERA schlechter. Allerdings gilt auch für die Beschäftigten: Je kooperativer der Prozess der ERAEinführung verläuft, desto besser wird ERA in der Regel bewertet. Kooperativität und Überschreiteranteil korrelieren negativ. Die beiden Einflüsse lassen sich jedoch nicht vollständig aufeinander reduzieren.

Betrachten wir die ERA-Bewertung einzelner Beschäftigtengruppen, so zeigt sich, dass nur wenige Gruppen mit ERA eher zufrieden sind: jüngere Beschäftigte, Vorgesetzte sowie Facharbeiter und ehemalige Zeitlöhner. Besonders unzufrieden sind die ehemaligen Leistungslöhner, gefolgt von den Beschäftigten der Verwaltungs- und Sekretariatsdienste. Auch höher qualifizierte technische und kaufmännische Angestellte und Angelernte sind überwiegend unzufrieden. Zufriedenheit mit ERA tritt somit nur dort auf, wo sich „Facharbeit" mit „Facharbeiter“ (im Zeitlohn) übersetzen lässt oder wo sich viele jüngere Beschäftigte finden, die weniger von der im Zeitverlauf gewachsenen Lücke zwischen tarifvertraglichen Mindestbestimmungen und betrieblicher Verdienstpraxis begünstigt waren.

Bemerkenswert ist, dass ERA keineswegs nur von Verlierern negativ bewertet wird. Unterschreiter, denen die ERA-Einführung einen monetären Zuwachs beschert, bewerten ERA im Mittel weder positiv noch negativ und ein großer Teil von ihnen fühlt sich durch ERA auch persönlich ungerecht behandelt. Nachgefragt, worin die „ungerechte Behandlung durch ERA“ bestehe, rangieren bei den Unterschreitern und den Überschreitern gleichermaßen die Angaben in der Dimension der Anerkennung („geringere Wertschätzung der Arbeit") vor den Angaben zur Unzufriedenheit mit den materiellen Effekten („Absenkung des Verdienstes“, „Verdienststeigerung zu gering"). Die Relevanz der Anerkennungsthematik zeigt sich nicht nur in der direkten Befragung der Beschäftigten, sondern ist inzwischen so offensichtlich, dass sie auch von nahezu allen interviewten Vertretern der Betriebs- und der Tarifparteien bestätigt wird. Der Zusammenhang zwischen Überschreiteranteil und ERA-Bewertung sollte deshalb nicht als Beleg dafür missverstanden werden, die Beschäftigten wären als ,homo oeconomicus" zureichend charakterisiert.

Das Merkmal Überschreiter signalisiert offenbar nicht nur vorübergehend geringere Verdienstzuwächse bzw. Reallohnverlust, sondern auch eine geringere Wertschätzung der gegenwärtigen und der vergangenen Leistungen. Und selbst wenn der Verdienst durch Ausgleichsbeträge gesichert bleibt, so markiert doch die Entgeltgruppe die eigentliche Position eines Beschäftigten im betrieblichen Statusgefüge. Geld und Einkommen sind sowohl in der Dimension der materiellen Interessen als auch in der Dimension der Anerkennung hoch bedeutsam. Eine adäquate Thematisierung des Verhältnisses von Lohn und Leistung kommt deshalb ohne eine Berücksichtigung der Anerkennungsdimension nicht aus (Schmidt 2005, S. 69).

Weshalb sind auch etliche Unterschreiter mit ERA unzufrieden? Zunächst dürften gemeinsame Belegschafts- oder Abteilungsdiskurse oder solidarische Orientierungen dafür sorgen, dass sich die Grenze persönlicher materieller Vor- und Nachteile nicht unverzerrt in der ERA-Bewertung ausdrückt. Zudem gibt es auch für Unterschreiter materielle Gründe für ERA-Unzufriedenheit. Mitunter wurden Hoffnungen auf größere materielle Zuwächse enttäuscht, oder die schrittweise Anhebung des Verdienstes auf das neue ERA-Niveau stieß auf Kritik. Ambivalent ist selbst eine deutliche Anhebung durch ERA, signalisiert sie in den Augen mancher doch zugleich, bisher unterbezahlt gewesen zu sein.

Sachlich fehlerhafte Aufgabenbeschreibungen bzw. Zuordnungen und zu niedrige Entgeltgruppen können sowohl bei Überschreitern als auch bei Unterschreitern Unzufriedenheit auslösen. Bedeutsam ist nicht zuletzt, dass im neuen System, anders als in der bisherigen analytischen Arbeitsbewertung, eine Beschränkung der Beschreibungen lediglich auf solche Aufgabenbestandteile zulässig ist, die die Wertigkeit der Tätigkeit prägen. Viele Beschäftigte sehen ihre Arbeit deshalb als mangelhaft wahrgenommen und ihren Beitrag als nicht adäquat gewürdigt an. Da die Konzentration auf wertigkeitsprägende Aufgabenmerkmale abstraktere Aufgabenbeschreibungen als bisher ermöglicht, werden bisweilen für die betroffenen Beschäftigten nicht nachvollziehbare Zuordnungen von Tätigkeiten zu einem Niveaubeispiel vorgenommen.
Überschreitern wie Unterschreitern dürfte nicht zuletzt der Bewertungsvorgang selbst aus zwei Gründen Unbehagen bereiten. Zum einen wird den Beschäftigten die Inkonsistenz zwischen dem auf Qualifikation und Beruflichkeit beruhenden Statussystem und dem anforderungsbezogenen Entgeltsystem durch die Erneuerung und damit zumindest vorübergehend faktische Radikalisierung des Anforderungsbezugs deutlich vor Augen geführt. Zum anderen entkleidet der Vorgang der anforderungsbezogenen Bewertung die Beziehung zum Betrieb selbst aller Illusionen und „professionellen Vorurteile“. Wenn anders als es bisher schien - weder die eigene Person noch Lebensleistung, Seniorität, Loyalität und eventuell noch nicht einmal die eigene Berufsidentität eine Rolle spielen, dann wird das von vielen Beschäftigten als Zumutung erfahren. Zur Disposition steht dann nicht nur der Wert der Ware Arbeitskraft, sondern der Charakter der Beziehung zum Betrieb selbst.

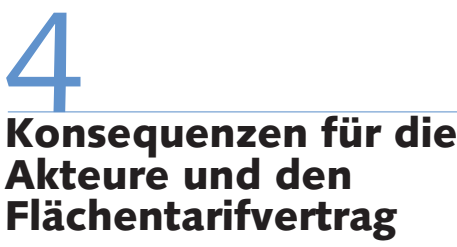

Die ERA-Einführung verlief in BadenWürttemberg konfliktreicher als erwartet. Sie hat die betrieblichen wie die überbetrieblichen Kollektivakteure erheblich strapaziert. Die Umsetzung hat Kraft gekostet und den meisten Akteuren, jedenfalls aus Sicht der Beschäftigten, einen Imageschaden eingebracht. Doch während die ERABewertung der Beschäftigten in starkem Maße von der Zahl der Überschreiter geprägt wird, determiniert Letztere die Bewertung der mit der ERA-Einführung befassten Kollektivakteure durch die Belegschaften nicht. Zwar hat sich die Meinung der Beschäftigten sowohl über das Management als auch über die Betriebsräte und die IG Metall im Kontext der ERA-Einführung verschlechtert, doch vor allem deren Bewertung seitens der Betriebsräte zeigt - in Abhängigkeit von deren Aktivitätsniveau - erhebliche zwischenbetriebliche Differenzen. Sowohl nach der direkten Befragung der Beschäftigten als auch in der Wahrnehmung der Betriebsräte richtet sich die Kritik der Beschäftigten primär an das betriebliche Management. $68 \%$ der Betriebsräte registrieren einen negativen Mei- 
nungswandel der Beschäftigten gegenüber dem Management. Bei den Betriebsräten selbst ist der Arbeitgeberverband Südwestmetall erster Adressat der Kritik. Dessen starke Einflussnahme machte ihnen zu schaffen und ihm werden auch zu einem erheblichen Teil die hohen Überschreiterquoten zugeschrieben. Drei Viertel der Betriebsräte bewerten den Verband negativer als zuvor.

\subsection{SÜDWESTMETALL}

Südwestmetall hat durch sein offensives Auftreten bei den Betriebsräten und Beschäftigten an Ansehen eingebüßt, nach eigener Darstellung jedoch nicht bei den Mitgliedsfirmen. Wegen ERA habe der Verband im Saldo keine Mitglieder verloren, eher einige hinzugewonnen. Für Mitgliederverluste, die es im Einführungszeitraum gab (2005 bis 2007:-15\%), werden andere Gründe genannt. Das Profil des Verbandes sei über Entgeltfragen hinaus geschärft worden. ERA habe eine Plattform geboten, eine breite und aufeinander abgestimmte Palette von Themen und Produkten in die Betriebe zu bringen, sich als kompetenter, offensiver Dienstleister zu präsentieren; man sei hier ,einen gewaltigen Schritt weiter gekommen"(Südwestmetall-Vertreter).

Kritik von Mitgliedsfirmen gibt es dennoch. Verärgert sind die Unternehmen nicht wegen der Kosten, diesbezüglich herrscht Zufriedenheit. Kritik gibt es wegen der Konflikte, die bei der ERA-Umsetzung auftraten. Die Friedensfunktion des Tarifvertrags, auf die die Firmen gesetzt hatten, konnte nicht gewährleistet werden. Das wird primär der IG Metall aber auch dem Arbeitgeberverband angelastet. Beiden wird vorgeworfen, die versprochene ruhige und einvernehmliche ERA-Umsetzung sei ausgeblieben. Dadurch wuchs nach Darstellung Südwestmetalls die Zahl der Skeptiker gegenüber Flächentarifverträgen in den eigenen Reihen. Ob daraus Konsequenzen gezogen werden, lässt der Verband derzeit offen. Die Ansage ist jedoch unmissverständlich: „Wenn wir ein zweites Mal Krieg führen müssen, wenn wir einen Flächentarifvertrag anwenden wollen, dann hat der für uns keine Funktion“ (Südwestmetall-Vertreter). Konflikte um Tarifverträge doppelt führen zu müssen, zunächst auf sektoraler, danach auf betrieblicher Ebene, gilt als unattraktive Perspektive, der gegebenenfalls der Verzicht auf eine Ebene vorzuziehen wäre. Es dürf- te keine Frage sein, dass dies die sektorale wäre. Auch wenn die Rechnung somit nicht ganz aufging, so geht Südwestmetall doch nicht geschwächt aus der ERA-Umsetzung hervor. Als Vorbild, an dem sich andere regionale Arbeitgeberverbände orientieren und dessen Beispiel Schule machen würde, fungiert Südwestmetall - auch in der eigenen Wahrnehmung - derzeit jedoch nicht.

\subsection{IG METALL}

Die IG Metall ging mit dem ERA-Projekt entgeltpolitisch wie organisationspolitisch ein deutlich höheres Risiko ein als Südwestmetall. Angesichts des Übergewichts an Überschreitern und der nicht geringen Zahl von Betrieben, in denen die Systemkosten von ERA unter den alten liegen dürften, hätte ERA leicht zu einem organisationspolitischen Debakel für die IG Metall werden können. Das ist es nicht geworden. Aber ihr Ansehen bei den Beschäftigten hat gelitten. Von einer Mehrheit wird sie schlechter als zuvor bewertet. Besonders verärgert sind die Angelernten bzw. die Leistungslöhner in der Produktion, d.h. die Beschäftigten in den traditionellen gewerkschaftlichen Hochburgen. Organisationspolitisch besteht das Dilemma für die IG Metall darin, dass mit ERA einerseits die traditionellen Bastionen verschreckt wurden und sie Mühe hat, dort ihren Rückhalt zu behalten; und dass sie andererseits bei ihrer Zukunftsklientel, den Angestellten, bisher nicht an Ansehen gewinnen konnte. Die Unzufriedenheit, die es gerade bei den gewerkschaftlich am besten organisierten Beschäftigtengruppen und - etwas verhaltener - auch bei den Betriebsräten gibt, hat allerdings nicht $\mathrm{zu}$ einer Welle von Gewerkschaftsaustritten geführt. Austritte wegen ERA wurden von Gewerkschaftseintritten etwa gleichen Umfangs kompensiert. ERA ist zu keinem organisationspolitischen Rückschlag geworden, den manche zwischenzeitlich befürchteten, zu einem Zugpferd der Mitgliedergewinnung allerdings ebenso wenig. Die phasenweise ausgesprochen negative Stimmung hat sich, wie die nachfolgenden Tarifbewegungen gezeigt haben, auch nicht in einer Einschränkung der Handlungsfähigkeit der IG Metall niedergeschlagen. Die Mobilisierungsfähigkeit hat nicht gelitten. Die Bereitschaft, sich an Warnstreiks zu beteiligen, war sogar eher stärker als ehedem. Die IG Metall ist, wie Betriebsräte und gewerkschaftliche Funktionäre nunmehr erleich- tert konstatieren, mit einem blauen Auge davon gekommen.

Mittelfristig könnte sich die organisationspolitische ERA-Bilanz für die IG Metall noch verbessern. Geliebt oder ungeliebt, ERA prägt die betrieblichen Entgeltstrukturen in einem seit Langem nicht mehr gekannten Maße und eröffnet damit Möglichkeiten für eine gewerkschaftliche, auf Tarifverträge aufbauende und durch sie geprägte betriebliche Entgeltpolitik. Betriebliche Entgeltkomponenten sind zudem vielfach in tarifliche überführt und oft über Ergänzungstarifverträge abgesichert worden. Voraussichtlich zukunftsfestere Entgeltstrukturen wurden implementiert. $\mathrm{Zu}$ dem ist im ERA-Prozess die Entgeltkompetenz der Betriebsräte wie der hauptamtlichen gewerkschaftlichen Funktionäre, d.h. die Kompetenz im „Kerngeschäft“ gewerkschaftlicher Politik, gegenüber den letzten Jahrzehnten deutlich gewachsen. Schließlich realisiert die "Zukunftsklientel“ der Gewerkschaft, die Angestellten, teilweise erstmals, dass ihre individuelle Entgeltfindung etwas mit Tarifverträgen zu tun hat. Anknüpfungspunkte für gewerkschaftliches Handeln wurden somit geschaffen. $\mathrm{Ob}$ sie genutzt werden (können), wird sich erst in den kommenden Jahren zeigen.

\subsection{TARIFSYSTEM}

ERA wurde von beiden Tarifparteien als Prüfstein für die Zukunft des Flächentarifvertrags verstanden. Ob die Prüfung bestanden wurde und der Flächentarifvertrag über ERA hinaus als Institution sicherer ist als zuvor, wird unterschiedlich gesehen. Während die Bezirksleitung der IG Metall den Flächentarifvertrag eindeutig als gestärkt und stabilisiert betrachtet, kann dies die Mehrheit der Betriebsräte, deren Einschätzung stark vom Verlauf des Einführungsprozesses und seinen Ergebnissen geprägt ist, derzeit nicht erkennen. Von einer Stärkung der Gestaltungskraft des Tarifvertrags durch ERA wollten am Ende des Einführungszeitraums nur wenige Betriebsräte sprechen (14\%), fast die Hälfte nimmt das Gegenteil an (45\%). Selbst wenn mitunter von einem gewissen, mittlerweile stattfindenden Wandel bei den Betriebsräten berichtet wird, ist das Image der Flächentarifverträge, die bisher als Instrument der Gewerkschaften zur Durchsetzung von Arbeitnehmerinteressen wahrgenommen wurden, bei den Betriebsräten eher angekratzt. 
Über die Bedeutung von ERA selbst hinaus macht die ERA-Einführung in Baden-Württemberg exemplarisch deutlich: Flächentarifverträge dürfen nicht (mehr) per se als Instrument der Gewerkschaften verstanden werden. Sie können zur Durchsetzung von Interessen der einen wie der anderen Seite dienen. Prinzipiell galt für Flächentarifverträge schon immer, dass sie von den jeweiligen Kräfteverhältnissen geprägte Kompromisslinien fixieren, in die Interessen beider Seiten eingehen. Der Flächentarifvertrag ist insofern ein Instrument, dessen Stimmung selbst umkämpft ist. Waren es traditionell die Gewerkschaften, die den Grundton der Stimmung festlegten, den Flächentarifvertrag als ihr Instrument deklarierten und auf eine strikte Umsetzung der Verträge drängten, hat sich diese Konstellation sichtbar verändert. Bei
ERA waren es die Arbeitgeber, die ihre Interessen in den tarifvertraglichen Bestimmungen so aufgehoben sahen, dass sie mit dem Ruf nach einer Eins-zu-eins-Umsetzung in die betriebliche Auseinandersetzung ziehen konnten (Bahnmüller/Schmidt 2006, 2007). Diese Konstellation, das wird von Südwestmetall unumwunden eingeräumt, gab es bisher so nicht: „Dass es der Arbeitgeber ist, der sich den Tarifvertrag holt, um seine Schutzposition durchzusetzen, ist relativ neu. Das war bisher das Mittel der Arbeitnehmer, auf das sie sich berufen konnten". Der interessenpolitische Gehalt von Tarifverträgen, so lehrt die Erfahrung, wandelt sich und er ist in jedem konkreten Einzelfall zu prüfen. Auch Arbeitgeber können Tarifverträge als ein Instrument zur Durchsetzung ihrer Interessen zu nutzen versuchen. Für Südwestme- tall war die ERA-Einführung ein Experiment. Die bisherigen Rollen zwischen den Tarifparteien wurden verkehrt. Der Verband setzte sich als Verfechter der Ordnungsfunktion des Tarifvertrages in Szene und gab die Losung aus, den Tarifvertrag buchstabengetreu umsetzen zu wollen. Erst im Umsetzungsprozess wurde deutlich, dass die Arbeitgeber überzogen hatten und sie unter dem Mantel der Ordnungspolitik den Degen der Verteilungspolitik führten. Quittiert wurde dies mit (verzögertem) Widerstand der IG Metall, der Betriebsräte und der Beschäftigten. Ein neues Modell des Umgangs mit Flächentarifverträgen, das in anderen Regionalverbänden von Gesamtmetall Schule machen und dauerhaft Erfolg versprechen würde, ließ sich wohl auch deshalb nicht etablieren.

\section{LITERATUR}

Bahnmüller, R./Schmidt, W. (2007): Auf halbem Weg. Erste Befunde zur ERA-Umsetzung in Baden-Württemberg, in: WSI-Mitteilungen 7, S. 358364

Bahnmüller, R./Schmidt, W. (2008): Der ERA und seine Umsetzung. Erfahrungen aus Baden-Württemberg, in: Bispinck, R. (Hrsg.): Verteilungskämpfe und Modernisierung. Aktuelle Entwicklungen in der Tarifpolitik, Hamburg, S. 78-118

Bispinck, R./Bahnmüller, R. (2007): Abschied vom Flächentarifvertrag? in: Bispinck, R. (Hrsg.): Wohin treibt das Tarifsystem, Hamburg, S. 9-28
IG Metall Baden-Württemberg/IG Metall Bildungsstätte Lohr (2004/2006): ERA-Wissen Handbuch I und II, Lohr

Schmidt, W. (2005): Industrielle Beziehungen, Interesse und Anerkennung. Plädoyer für eine duale Perspektive, in: Industrielle Beziehungen 12, S. 51-73

Walton, R. E./McKersie, R. B. (1965): A Behavioral Theory of Labor Negotiations. An Analysis of a Social Interaction System, New York 